\title{
Nanoscale Infrared Spectroscopy, Technique and Applications
}

\author{
K. Kjoller, ${ }^{*}$ D. Cook* R. Shetty* and C. Prater* \\ *Anasys Instruments, 121 Gray Ave. Suite 100, Santa Barbara, CA 93101
}

Conventional IR microscopy is widely used in a range of research but is limited from applications where feature sizes are approximately 10 microns and lower. This is due to the diffraction limit of the mid IR illumination which limits the resolution to roughly three times the wavelength. ${ }^{1}$ Atomic force microscopy (AFM) has been very successful in visualizing the structure of samples at the nanoscale. Researchers have long recognized that combining the nanoscale resolution of the AFM with the chemical identification of IR spectroscopy would provide a powerful analytical technique to chemically characterize regions of the sample.

Several AFM probe-based techniques have been developed in an attempt to surpass the diffraction limit of conventional IR measurements. They include both traditional near field scanning optical microscopy (NSOM) as well as aperatureless near field scanning microscopy (ANSOM). The ANSOM technique has demonstrated impressive lateral resolution ${ }^{2}$ but has not produced rich spectra that can be used to characterize a broad range of vibrational resonances. Additionally the ANSOM technique suffers from a limited signal to noise restricting analysis to specific sample classes. Other techniques developed to extend the resolution of IR spectroscopy are based on measuring the local temperature rise from spectral absorption through the use of AFM cantilevers integrated with conventional Fourier Transform Infrared (FTIR) spectrometers. ${ }^{3}$ These approaches allow broader spectrum measurement than near-field approaches, but the spatial resolution is typically limited due to thermal diffusion to the scale of many microns.

We have developed and utilized a novel lab-based instrument that combines AFM and infrared spectroscopy to enable chemical characterization of samples at sub-micron scales. The instrument makes use of a technique called photothermal induced resonance (PTIR). ${ }^{4}$ PTIR is accomplished by using a traditional AFM probe to sense the rapid local expansion of a sample that absorbs light from a pulsed IR source. As shown in figure 1, the sample is illuminated with a pulsed, tunable IR light source in a configuration similar to the attenuated total reflection (ATR) technique used in conventional IR spectroscopy. When the IR illumination is at a wavenumber which is an absorption band of the sample, it creates a rapid thermal expansion wave that excites resonant oscillations of the AFM cantilever. By measuring the amplitude of the cantilever vibrations as a function of the IR source wavelength, a local IR absorption spectrum can be created. The IR source is continuously tunable from 3600 to $1200 \mathrm{~cm}^{-1}$. This covers a major portion of the mid-IR including important $\mathrm{CH}$, $\mathrm{NH}$ and $\mathrm{CO}$ bands, as well as carbonyl and amide I/II bands.

We have used this novel technique to image and chemically identify a variety of polymer samples. Figure 2 shows measurements performed on a blend of polycarbonate (PC) and acrylonitrile butadiene styrene (ABS) typically used as an impact modified polymer. The two components could be identified by the shift in the $\mathrm{CH}$ stretch peak from 2920 to $2970 \mathrm{~cm}^{-1}$ as the tip moves from PC to ABS domains.

\section{References}

[1] See for example: http://www.thermo.com/eThermo/CMA/PDFs/Articles/articlesFile_7208.pdf

[2] B. Knoll et al., Nature 399 (6732), 134-137 (1999).

[3] A. Hammiche et al., Spectroscopy 19 (2), 20-+ (2004). 
[4] A. Dazzi et al., Optics Letters 30 (18), 2388-2390 (2005).

[5] This work was supported in part by NIST-ATP 70NANB7H7025 and NSF-SBIR 0750512.

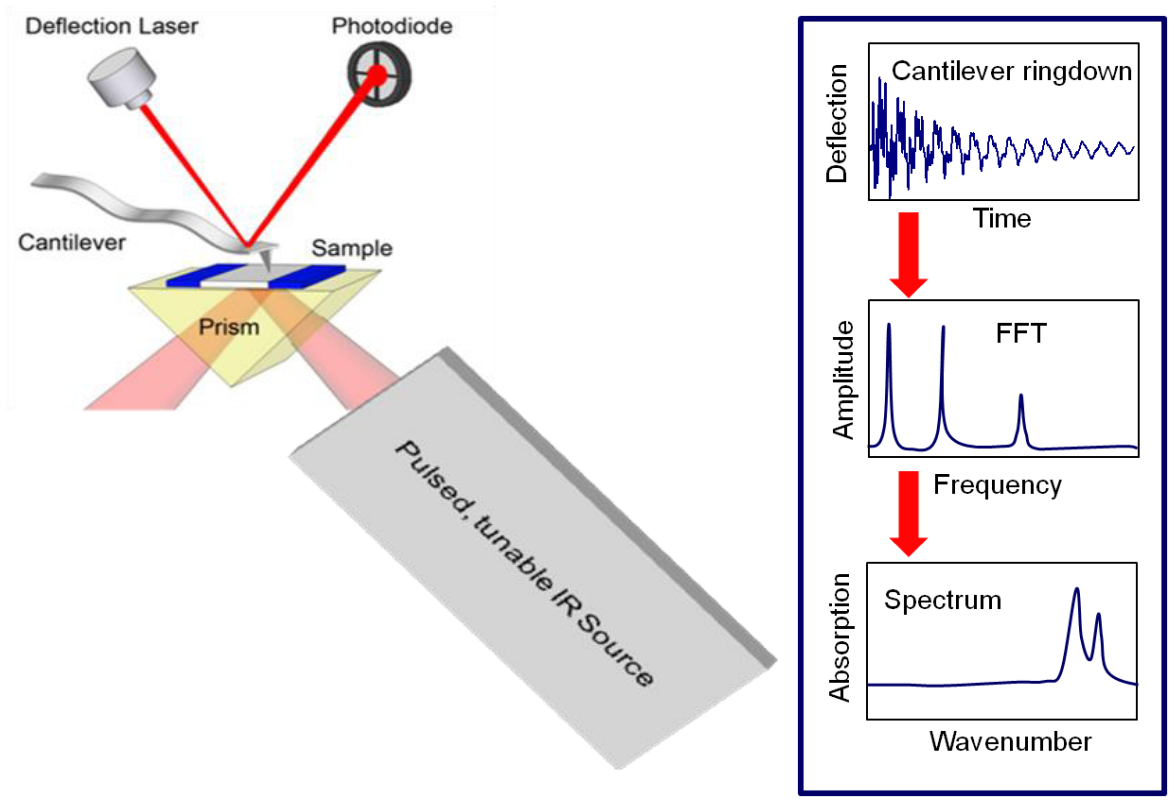

FIG. 1. This instrument uses a pulsed, tunable IR source to excite molecular resonances in the sample. Absorption of IR radiation by the sample leads to a rapid thermal expansion that excites resonant oscillations of the cantilever. The oscillations can be analyzed as a function of the source wavelength to create local absorption spectra.

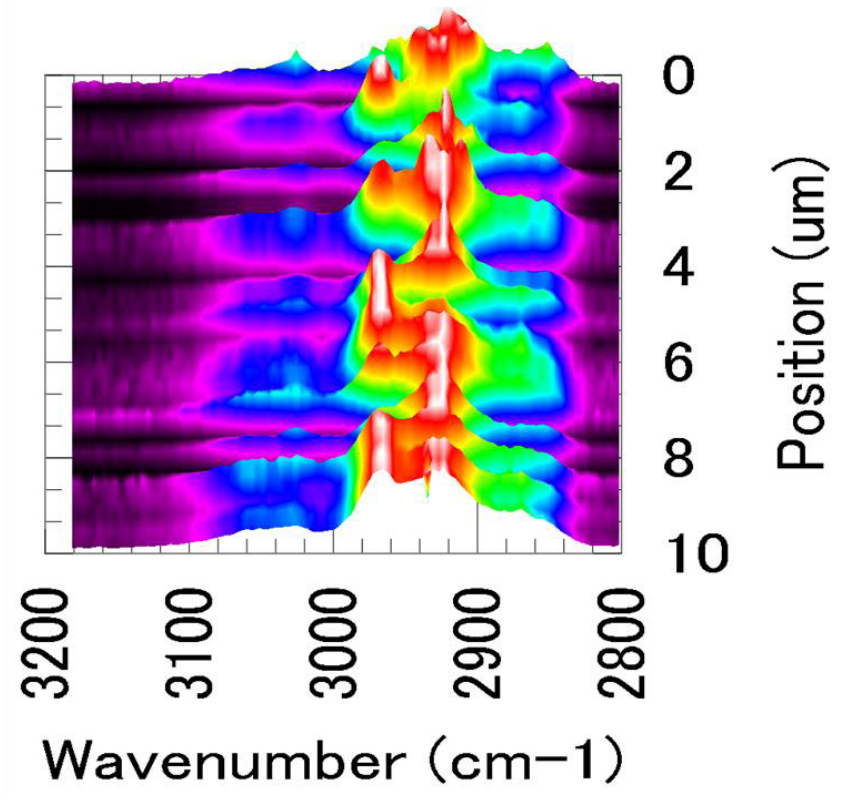

FIG. 2. A line spectral map of a blend of polycarbonate (PC) and acrylonitrile butadiene styrene (ABS). The shift in the position of the highest $\mathrm{CH}$ peak from $\sim 2920$ to $2970 \mathrm{~cm}-1$ indicates the regions of $\mathrm{ABS}$ from the regions of $\mathrm{PC}$, respectively. 